\title{
Optimized-Based Stabilization of Constrained Nonlinear Systems: A Receding Horizon Approach *
}

\author{
De-feng $\mathrm{He}^{*}, \mathrm{Li} \mathrm{Yu}{ }^{*}, \mathrm{Xiu}-$ lan Song* \\ * School of Information Engineering, Zhejiang University of \\ Technology, Hangzhou, Zhejiang, 310023 P.R.China (e-mail: \\ hdfzj@zjut.edu.cn, lyu@zjut.edu.cn,songxl@zjut.edu.cn).
}

\begin{abstract}
The problem of stabilizing constrained nonlinear systems meanwhile optimizing some performances is investigated in this paper. The tool of weak control Lyapunov functions is introduced to construct a tuning Sontag's controller where some adjustable parameters are optimized with respect to given performances in way of receding horizon fashion. Two algorithms are presented and the corresponding closed-loop systems with input constraints are proven to be stable in some regions by using the LaSalle's theorem and weak control Lyapunov functions. Moreover, the inverse optimality result of the controller is achieved. Finally, an open-loop unstable example is given to illustrate the effectiveness of the results obtained here.
\end{abstract}

Keywords: Input-affine nonlinear systems, weak control Lyapunov functions, receding horizon control, stabilization, inverse optimality.

\section{INTRODUCTION}

Control problems generally involve the both issues of stabilization and optimality of some given performances for constrained nonlinear systems, which have been the subject of intense research work in the past few years. The notion of control Lyapunov functions (CLFs) (Sepulchre et al.1997) has been a good tool to study the problem of stabilization of unconstrained nonlinear systems. For input-affine nonlinear systems, a stabilizing controller based on the famous Sontag's formula (Sontag,1989) can be constructed when a CLF of the system is found. This controller not only asymptotically stabilizes the system, but also has inverse optimality result in case of unconstraint. Moreover, the controller is globally continuous with the small control property (Sontag,1989). Consequently, several controllers based on the Sontag's formula have been proposed for unconstrained nonlinear affine systems (See e.g. El-Farra et al.2003; Lin,1991; Kokotovic et al.2001). Nevertheless, the constraints and optimality of given performances are not directly considered in design of the controller. In fact, the performance of this controller almost relies on the chosen CLFs for the controlled system, that is, there are not degrees of freedom of the controller for a chosen CLF.

On the other hand, receding horizon control (RHC) scheme has received much interest in academic community in the last years since it provides an excellent theoretical framework for handling explicitly constraints and optimality of given performances (Mayne et al.2000; Qin et al.2003). In

\footnotetext{
* This work is supported by the Natural Science Foundation of China under Grant 60904040, Specialized Research Fund for Doctoral Program of Higher Education of China under Grant 20093317120002 and Natural Science Foundation of Zhejiang Province under Grant Y1100911.
}

general, RHC law is obtained by solving, on-line, a finite horizon optimal control problem at each sampling time using the current state as the initial condition. In order to achieve the stability results of RHC closed-loop systems, the notion of CLFs has been introduced to the synthesis of the RHC scheme in recent years. For example, Primbs et al. (2000) generalized pointwise min-norm Sontag's controllers in way of RHC form for unconstrained nonlinear systems. By combination of variation of Sontag's formula, McConley et al. (2000) presented a stable scheduling procedure for nonlinear systems; Bloemen et al. (2002) for bilinear systems; El-Farra et al. (2004) for hybrid systems and De-feng et al. (2008) gave constructive RHC schemes for constrained systems. These approaches can be also seen as a way to consider constraints and optimality of given performances in the synthesis of controllers based on the rigid CLFs Sontag's formula. However, to the best of our knowledge, it is not easy to construct a rigid CLF for constrained nonlinear systems.

In this paper, the receding horizon approach is extended to design stabilized Sontag's controllers for nonlinear affine systems subject to input constraints. The concept of weak CLFs (WCLFs) is introduced and used to construct an adjustable Sontag's formula. Then two RHC-based algorithms are formulated to compute the adjustable parameters in the controller by minimizing a finite horizon optimal control problem. Furthermore, the asymptotic stability and inverse optimality properties of the closed-loop system are achieved by using the LaSalle's theorem (Khalil,2002) and property of WCLFs, regardless of the optimization problem. Finally, an open-loop unstable example is used to illustrate the effectiveness of the results obtained here.

The paper is organized as follows: Section 2 describes the controlled system and defines the notion of WCLFs. 
Two RHC-based algorithms and corresponding theoretical results are presented in Section 3. An example is given and simulation results are discussed in Section 4 and Section 5 contains the conclusion.

Notation: The symbol $R_{+}$denotes the set of all nonnegative real numbers. For any vector $x, x^{\prime}$ is its transpose and $\|x\|$ its Euclidean norm. The notation $L_{f} V$ is standard Lie derivative of a scalar function $V(\cdot)$ along the vector function $f(\cdot)$. The solution of a system at time $t$ from the initial state $x\left(t_{0}\right)$ at initial time $t_{0}$ for an input signal $u$ is denoted by $x(t)=\varphi\left(t ; x\left(t_{0}\right), u\right)$.

\section{SYSTEM DESCRIPTION}

Consider an input-affine nonlinear system described by

$$
\dot{x}(t)=f(x(t))+g(x(t)) u(t), \quad t \geq 0, x(0)=x_{0},
$$

where state vector $x(t) \in R^{n}$, control vector $u(t) \in R^{m}$, and all entries of the vector $f$ and the $n \times m$ matrix $g$ are known smooth functions on $R^{n}$. The system is subject to constraint on control actions given below

$$
u(t) \in U \subseteq R^{m}, \quad t \geq 0,
$$

where $U$ is a compact set containing the origin. Without loss of generality, it is assumed that the origin is an equilibrium point of the system and the state is available for feedback. Throughout the paper, we also assume that the system frees uncertainty and its solution $\varphi(\cdot ; x(0), u)$ is continuous and unique for any initial state $x_{0}$ in the region of interest and any piecewise continuous and right continuous input function $u(\cdot): R \rightarrow U$.

Definition 1. A positive definite function $V: R^{n} \rightarrow R_{+}$ is called a weak control Lyapunov function (WCLF) for system (1) if it is smooth, radially unbounded in $x$ and such that

$$
L_{g} V(x)=0 \Rightarrow L_{f} V(x) \leq 0, \quad \forall x \neq 0,
$$

where $L_{g} V(x)=\left[L_{g_{1}} V(x) \ldots L_{g_{m}} V(x)\right]$ and $g_{i}(x), i=$ $1 \ldots m$, is the $i$ th column of the matrix $g(x)$.

If a WCLF, $V(x)$, for system (1) exists, a tuning formula closing to the Sontag's one can be constructed

$$
h(x, \mu)= \begin{cases}-\kappa(x) \beta(x)^{\prime}, & \text { if }\|\beta(x)\| \neq 0 \\ 0, & \text { if }\|\beta(x)\|=0\end{cases}
$$

where $\alpha(x)=L_{f} V(x), \beta(x)=L_{g} V(x)$, real number $\mu>0$ is an adjustable parameter and

$$
\kappa(x, \mu)=\frac{\alpha(x)+\mu \sqrt{\alpha(x)^{2}+\|\beta(x)\|^{4}}}{\|\beta(x)\|^{2}}
$$

Let $u=h(x, \mu)$. Clearly, the adjustable parameter, $\mu$, in (4) increases the degrees of freedom of the controller and can be determined by minimizing some given performances. In this case, the controller still merits some good properties from Sontag's controllers.

\section{RHC-BASED SONTAG'S CONTROLLER}

The RHC law with initial state $x$ at time $t$ is obtained by computing on-line an open-loop optimal control problem over time interval $[t, t+T]$ and is then applied to the plant over time $[t, t+\delta)$, where $\delta>0$ is a sufficiently small sampling period. At next sampling time, the optimizing process is repeated with new measurements. Hence, the RHC-based Sontag's controller is formulated as (for simplicity, let the control and prediction horizons satisfy $T_{c}=T_{p}=T$.):

$$
\begin{array}{ll}
\min _{\mu(t)>0}\left\{J(x, u, t)=\int_{t}^{t+T} l(x(s), u(s)) d s\right\} \\
\text { s.t. } \quad \dot{x}(s)=f(x(s))+g(x(s)) u(s), \\
\quad u(s)=h(x(s), \mu(t)), \\
\quad u(s) \in U, \quad t \leq s<s+T
\end{array}
$$

where stage cost $l(x, u)$ is a nonnegative definite function on $x$ and positive definite function on $u$. The designer can choose the different stage cost $l(x, u)$ and horizon length $T$ to meet different control performances.

If the problem (5) is feasible at each time, the RHC-based Sontag's controller $u^{r h c}(\cdot)$ can be defined by

$$
u^{r h c}(s)=h\left(x(s), \mu(t)^{*}\right), \quad \forall s \in[t, t+\delta)
$$

where $\mu(t)^{*}$ is the solution to (5) at the current time $t$. The closed-loop system is then represented as

$$
\dot{x}(s)=f(x(s))+g(x(s)) u^{r h c}(s), \quad \forall s \in[t, t+\delta)
$$

Definition 2. If subset $S \subseteq R^{n}$ is an invariant set of the closed-loop system (7) and feedback law (6) satisfies the constraint (2) for all $x \in S$, then $S$ is called an operation region of the system $(7)$.

\section{Algorithm 1: (optimal design)}

(1) Given the stage cost $l(x, u)$, horizon length $T$, and sufficiently small sampling period $\delta>0$.

(2) Construct the controller, $h(x, \mu)$, by using the equation (4).

(3) Compute on-line the parameter $\mu(t)$ in $h(x, \mu)$ for the problem (5).

(4) Implement $u(s)=h\left(x(s), \mu(t)^{*}\right)$ for $t \leq s<t+\delta$, set $t=t+\delta$ and go back to (3).

In the sequent, we present the stability results for the closed-loop system (7) with Algorithm 1.

Theorem 1. Let $V(x)$ be a WCLF of the system (1) and set $S_{1}=\left\{x \in R^{n}: \alpha(x)=0, \beta(x)=0\right\}$. Assume that the closed-loop system (7) has only trivial solution in set $S_{1}$. Then there exists a nonempty set $\Theta(c) \subseteq R^{n}$

$$
\Theta(c)=\left\{x \in R^{n}: V(x) \leq c, \quad c>0\right\}
$$

such that for all $x(0) \in \Theta(c), h\left(x, \mu^{*}\right) \in U$ and the system (7) with Algorithm 1 is asymptotically stable in an operation region $\Theta(c)$. Moreover, if the inequality (9) holds,

$$
\mu(t)^{*} \sqrt{\alpha(x(t))^{2}+\|\beta(x(t))\|^{4}} \geq \alpha(x(t)), \quad \forall t \geq 0
$$

the control law (6) is optimal for the cost functional:

$$
\left\{\begin{array}{l}
J=\int_{0}^{\infty}\left[L\left(x(t), \mu(t)^{*}\right)+\frac{u(t)^{\prime} u(t)}{2 p\left(x(t), \mu(t)^{*}\right)}\right] d s \\
L\left(x(t), \mu(t)^{*}\right)=\frac{1}{2} p\left(x(t), \mu(t)^{*}\right) \beta(x(t)) \beta(x(t))^{\prime}-\alpha(x(t))
\end{array}\right.
$$

where $\mu(t)^{*}$ is determined by Algorithm 1 and 


$$
p\left(x, \mu^{*}\right)=\left\{\begin{array}{ll}
\kappa\left(x, \mu^{*}\right), & \text { if }\|\beta(x)\| \neq 0 \\
0, & \text { if }\|\beta(x)\|=0
\end{array} .\right.
$$

Proof. With Algorithm 1, we obtained an optimizing solution, $\mu(t)^{*}$, to problem (5) at time instance $t$, which yields a control law $h\left(x(s), \mu(t)^{*}\right)$. Since the origin is the interior of $U$ and $h\left(x(s), \mu(t)^{*}\right)$ is continuous in $R^{n}$, we can always find a constant $c_{1}>0$ such that $h\left(x(s), \mu(t)^{*}\right) \in U$ for all $x \in \Theta\left(c_{1}\right)$. Let $c \in\left(0, c_{1}\right]$ and define the region $\Theta(c)$ in form of (8). Then the input constraint is fulfilled for all $x(0) \in \Theta(c)$.

Differentiating $V(x)$ along a trajectory of the closed-loop system in (7), we can obtain

$$
\begin{aligned}
\dot{V}(x) & =\alpha(x)+\beta(x) u^{r h c} \\
& = \begin{cases}-\mu^{*} \sqrt{\alpha(x)^{2}+\|\beta(x)\|^{4}}, & \text { if }\|\beta(x)\| \neq 0(11) \\
\alpha(x), & \text { if }\|\beta(x)\|=0\end{cases}
\end{aligned}
$$

for all $x \in \Theta(c)$ and $\mu^{*}>0$. With the properties of WCLFs, equality (11) yields

$$
\dot{V}(x) \leq 0, \quad \forall x \in \Theta(c), \mu^{*}>0
$$

Based on standard Lyapunov arguments, inequality (12) implies that any trajectory of the system (7) starting in $\Theta(c)$ still remains in $\Theta(c)$ and the equilibrium point of the system (7) is stable. Thus the region $\Theta(c)$ is an invariant set of the closed-loop system and is an operation region of the closed-loop system by Definition 2. Furthermore, the WCLF $V(x)$ satisfies that

$$
\dot{V}(x)=0 \Leftrightarrow \alpha(x)=0, \beta(x)=0
$$

for all $x \in \Theta(c)$ and $\mu^{*}>0$. With the assumption that the closed-loop system (7) has only trivial solution in set $S_{1}$, the closed-loop system (7) is then asymptotically stable in its operation region $\Theta(c)$ by the LaSalle's theorem (Khalil,2002).

In order to prove that the control law (6) is optimal for the cost functional (10), we need examine the non-negativity of the cost functional with respect to $\mu^{*}$ at each sampling time $t \geq 0$.

Consider $\|\beta(x)\|=0$. Then by using the properties of WCLFs, the stage cost $L$ in (10) is equal to

$$
L\left(x, \mu^{*}\right)=-\alpha(x) \geq 0
$$

Again for $\|\beta(x)\| \neq 0$, it is obtained that

$$
\begin{aligned}
L\left(x, \mu^{*}\right) & =\frac{1}{2} p\left(x, \mu^{*}\right) \beta(x) \beta(x)^{\prime}-\alpha(x) \\
& =\frac{1}{2}\left[\mu^{*} \sqrt{\alpha(x)^{2}+\|\beta(x)\|^{4}}-\alpha(x)\right]
\end{aligned}
$$

By instituting (9) into (15) yields

$$
L\left(x, \mu^{*}\right) \geq 0, \quad \forall x \in \Theta(c), \mu^{*}>0
$$

at each sampling time $t \geq 0$. Then from (14) and (16), we have that the cost functional in (10) is meaningful for all $x$ in $\Theta(c)$.

Let $v(t)=u(t)-h\left(x(t), \mu(t)^{*}\right)$ and be instituted into (10), we obtain that

$$
\begin{aligned}
J= & \int_{0}^{\infty}\left\{\frac{1}{2} p\left(x(t), \mu(t)^{*}\right) \beta(x(t)) \beta(x(t))^{\prime}-\right. \\
& \alpha(x(t))+\frac{1}{2 p\left(x(t), \mu(t)^{*}\right)} . \\
& {\left.\left[v(t)+h\left(x(t), \mu(t)^{*}\right)\right]^{\prime}\left[v(t)+h\left(x(t), \mu(t)^{*}\right)\right]\right\} d s } \\
= & \int_{0}^{\infty}\left\{p\left(x(t), \mu(t)^{*}\right) \beta(x(t)) \beta(x(t))^{\prime}-\alpha(x(t))+\right. \\
& \left.\frac{v(t)^{\prime} v(t)}{2 p\left(x(t), \mu(t)^{*}\right)}-\beta(x(t)) v(t)\right\} d s \\
= & \int_{0}^{\infty}\left\{\frac{v(t)^{\prime} v(t)}{2 p\left(x(t), \mu(t)^{*}\right)}-\alpha(x(t))-\beta(x(t)) u(t)\right\} d s \\
= & \int_{0}^{\infty}\left\{\frac{v(t)^{\prime} v(t)}{2 p\left(x(t), \mu(t)^{*}\right)}-\dot{V}(x(t))\right\} d s \\
= & V(x(0))-\lim _{T \rightarrow \infty} V(x(t))+\int_{0}^{\infty} \frac{v(t)^{\prime} v(t)}{2 p\left(x(t), \mu(t)^{*}\right)} d s
\end{aligned}
$$

Since the closed-loop system is asymptotically stable in an operational region $\Theta(c)$, equation (17) results in

$$
J=V(x(0))+\int_{0}^{\infty} \frac{v(t)^{\prime} v(t)}{2 p\left(x(t), \mu(t)^{*}\right)} d s
$$

It is apparent that the cost functional (18) is minimal if $v(t)=0$, i.e. $u(t)=h\left(x(t), \mu(t)^{*}\right)$. Then the control law (6) is optimal for the cost functional (10).

This completes the proof of Theorem 1.

Clearly, the size of the invariant set $\Theta(c)$ depends on the constraint (2) and the value of $\mu^{*}$. However, it is not an easy to exactly compute $\Theta(c)$, even if the controlled system is linear. Hence, in order to derive the largest invariant set of $\Theta(c)$, we may make the static programming problem offline

$$
\begin{aligned}
& \max _{\mu>0} c \\
& \text { s.t. } h(x, \mu) \in U, \forall x \in \Theta(c)
\end{aligned}
$$

If a suitable pair $(\tilde{c}, \tilde{\mu})$ is found in this way, it determines a region $\Theta(\tilde{c})$ in form of (8), where Theorem 1 is true. On the other hand, Algorithm 1 requires the optimality of the solution. However, the computation of such solution is highly expensive due to its non-convexity nature. This makes difficulties of implementation of Algorithm 1 for fast sampling applications. Hence, the theoretical and practical properties under suboptimality of the obtained solution should be more carefully considered. In what follows, we assume that the pair $(\tilde{c}, \tilde{\mu})$ has been identified and then a suboptimal version corresponding to Algorithm 1 is developed.

\section{Algorithm 2: (suboptimal design)}

(1) Pick a number $\rho \in(0,1]$.

(2) Given the stage cost $l(x, u)$, horizon length $T$, and sufficiently small sampling period $\delta>0$.

(3) Construct the controller $h(x, \mu)$ in (4) and compute off-line the largest invariant set $\Theta(\tilde{c})$ in (19).

(4) Compute on-line the parameter $\mu(t)$ in $h(x, \mu)$ for the problem (5) such that, with initial guess $\mu=\tilde{\mu}$

$$
J(x, u(x, \mu(t)), t) \leq \rho J(x, u(x, \tilde{\mu}), t) .
$$

(5) Implement $u(s)=h\left(x(s), \mu(t)^{*}\right)$ for $t \leq s<t+\delta$, set $t=t+\delta$ and go back to $(4)$. 
Similarly, Algorithm 2 has the same stability and inverse optimality results to Algorithm 1.

Theorem 2. Under the assumption of Theorem 1, the closed-loop system (7) with Algorithm 2 is asymptotically stable in the operation region $\Theta(\tilde{c})$. Moreover, if the inequality (9) holds, the control law (6) with Algorithm 2 is optimal for the cost functional (10).

Proof. The process of the proof is similar to that of Theorem 1 and is thus omitted.

Remark 1: It is seen in (11) that the parameter, $\mu$, is likely to play the role of 'decaying rate' of systems to be controlled and hence, impacts significantly on the degree of stability of the closed-loop system. This implies that we can derive, to a certain extent, a prescribed rate of decaying of the controlled systems via tuning the value of $\mu$. Moreover, we can choose different $\mu$ to achieve satisfactory control performance, without loss of the closed-loop stability. This is the key idea for the both algorithms presented here.

Remark 2: It is hoped that the stability properties of closed-loop systems are decoupled from the specifications of control performances for optimized-based control. The both algorithms presented here have this merit, that is, the closed-loop stability with both algorithms is independent of the choice of such $T$ and $l(x, u)$ as performance parameters in its operational regions. From a practical point of view, the user can adjust on-line these parameters to achieve certain goals while, at the same time, does not require any concern of losing stability. Clearly, this merit is well-desired to such situation that the amount of time available for on-line computation is not constant or is very short. Note also that if the system (1) frees constraints, Algorithm 1 and 2 guarantee globally asymptotic stability of the closed-loop system, respectively.

\section{SIMULATION EXAMPLE}

With the difficulties of computing a global solution to the optimal problem (5), we here employ the suboptimal version to illustrative the main ideas in the paper. Consider the $2 d$ nonlinear oscillator in Primbs et al. (2000):

$$
\left\{\begin{array}{l}
\dot{x}_{1}=x_{2} \\
\dot{x}_{2}=-x_{1}\left(\frac{\pi}{2}+\arctan \left(5 x_{1}\right)\right)-\frac{2.5 x_{1}^{2}}{1+25 x_{1}^{2}}+4 x_{2}+3 u
\end{array}\right.
$$

where input variable $u$ is subject to the box constraint $[-8,8]$ and the origin is an unstable equilibrium point of its open-loop system. It is very difficult in finding a CLF for the system (21). So, select a quadratic function $V(x)$ (also given in Primbs et al. (2000)) as a WCLF of the system

$$
V(x)=\frac{\pi}{2} x_{1}^{2}+x_{2}^{2}
$$

Then we construct the Sontag's controller by using equations (4) and compute the largest operation region $\Theta(\tilde{c})$ in $(19)$ with $(\tilde{c}, \tilde{\mu})=(18.2,0.08)$ (See ellipse curve in Fig. 1).

In the simulation running, the parameters in the optimal problem (5) are chosen as $T=0.3 \mathrm{~s}$ and

$$
l(x, u)=5 x_{1}^{2}+x_{2}^{2}+u^{2}
$$

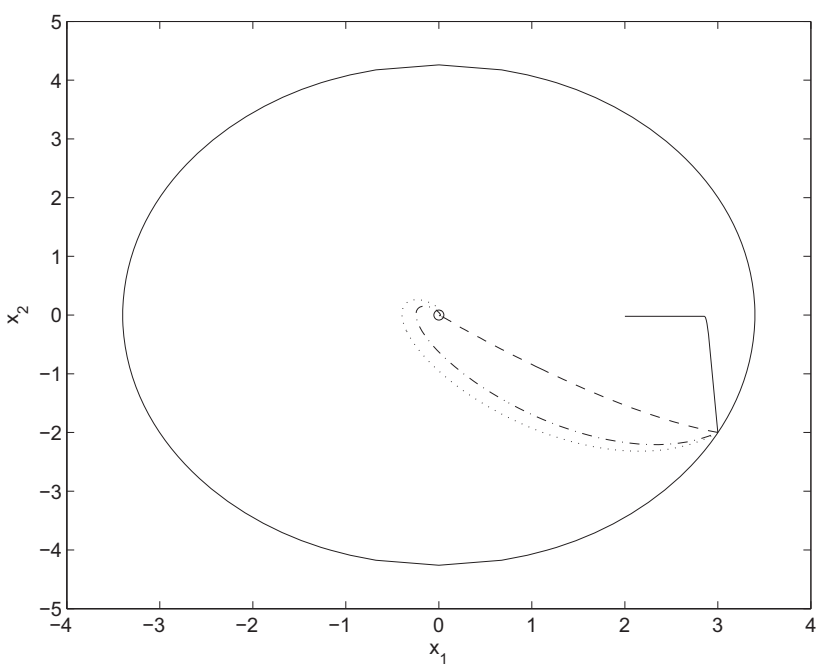

Fig. 1. Phase portraits of Sontag's controller with $\mu=0.08$ (dotted), $\mu=1$ (solid), and based on RHC with $T=1 \mathrm{~s}$ (dashed), $T=0.3 \mathrm{~s}$ (dash-dot)

The simulation is processed in discrete-time with a sampling interval $\delta=0.05 \mathrm{~s}$ and a simulation time of $7.5 \mathrm{~s}$. A few trajectories associated with different controllers applied the unstable constrained system (21) from the same initial condition $\left(x_{1}, x_{2}\right)=(3,-2)$ are shown in Figs. 1-4. The solid curve in Fig. 1, corresponding to the standard Sontag's controller (i.e., $\mu=1$ ), does not reach the origin until the simulation time is more than $150 \mathrm{~s}$. The corresponding states and input profiles are shown by the solid lines in Figs. 2-4, respectively. Note that for uniformities of figures, Figs. 2-4 are pictured only the parts of the profiles in the simulation time. Using the algorithms proposed in this paper, however, the plant not only can be stabilized asymptotically to the origin but also achieves a better control performance (see dash-dot lines in Figs. 1-4). It is important to point that, for any initial state in the operational region $\Theta(\tilde{c})$, one could try to tune the horizon length further in order to ease the online computational burden or derive better control performance, without any concern of losing stability of the closed-loop system. Taking an extreme case when $T \rightarrow 0$, we directly choose the initial guess, i.e., the Sontag's controller with $\mu=0.08$ s as a solution of the optimization problem, the computational burden of which is minimal. The dotted lines in Figs. 1-4 show this result. On the other hand, if the available time for the online computation is sufficient, we increase the horizon length to $T=1$ s and achieve a better performance. This result is presented by dashed lines in Figs. 1-4. Finally, it is clearly seen that the obtained controllers here do not violate the control constraint. By contraries, the standard Sontag's controller violates the constraint since its maximal control actions are more than the upper bound of input at the previous times.

\section{CONCLUSION}

By using receding horizon approach, we proposed an optimized-based stabilization control of input-affine nonlinear systems subject to input constraints. Two RHCbased algorithms were presented, where weak control Lyapunov functions were exploited to construct a tuning 


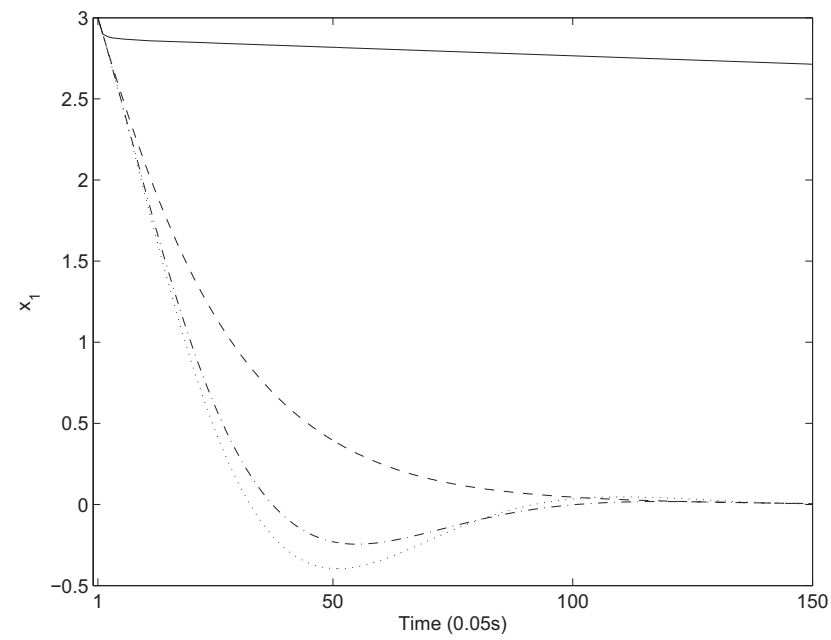

Fig. 2. Closed-loop state $x_{1}$ profiles of Sontag's controller with $\mu=0.08$ (dotted), $\mu=1$ (solid), and based on RHC with $T=1 \mathrm{~s}$ (dashed), $T=0.3 \mathrm{~s}$ (dash-dot)

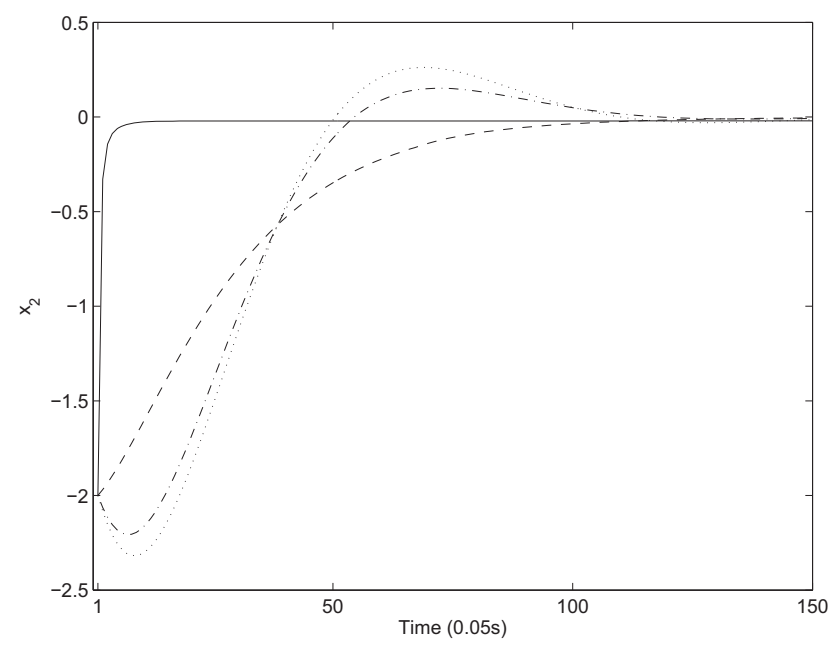

Fig. 3. Closed-loop state $x_{2}$ profiles of Sontag's controller with $\mu=0.08$ (dotted), $\mu=1$ (solid), and based on RHC with $T=1$ s (dashed), $T=0.3 \mathrm{~s}$ (dash-dot)

Sontag's controller and some adjustable parameters were optimized in fashion of receding horizon approach, with respect to given performances. The asymptotic stability and inverse optimality results of closed-loop systems with both algorithms were achieved by the properties of weak CLFs and the LaSalle's theorem. Finally, a numerical simulation on a $2 \mathrm{~d}$ nonlinear oscillator demonstrated the effectiveness of algorithms proposed here. Further research effort could involve studying robustness properties of the constrained closed-loop systems with the both algorithms presented here.

\section{REFERENCES}

H. Bloemen, M. Cannon and B. Kouvaritakis. An interpolation strategy for discrete-time bilinear MPC problems. IEEE Trans. Autom. Contr., 45(5):775-778, 2002.

H. De-feng, J. Hai-bo and Z. Tao. Constructive model predictive control for constrained nonlinear systems. Optimal Control Applicaitons \& Methods, 29:467-481, 2008.

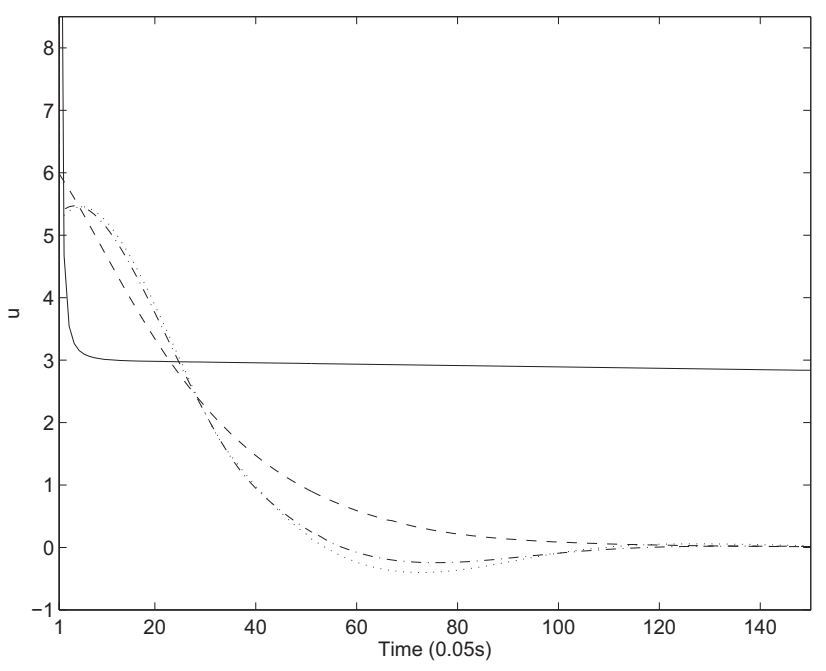

Fig. 4. Input profiles of Sontag's controller with $\mu=0.08$ (dotted), $\mu=1$ (solid), and based on RHC with $T=1 \mathrm{~s}$ (dashed), $T=0.3 \mathrm{~s}$ (dash-dot)

N.H. El-Farra, P. Mhaskar and P.D.Christofides. Uniting bounded control and MPC for stabilization of constrained linear systems. Automatica, 40(1):101-110, 2004.

N.H. El-Farra and P.D.Christofides. Bounded robust control of constrained multivariable nonlinear processes. Chem. Eng. Sci., 58(13):3025-3047, 2003.

H.K. Khalil. Nonlinear systems (2nd edition). New York: Prentice Hall, 2002.

P. Kokotovic and M. Arcak. Constructive nonlinear control: a historical perspective. Automatica, 37:637662, 2001.

Y. Lin and E.D. Sontag. A universal formula for stabilization with bounded controls. System \& control Letters, 16(6):393-397, 1991.

D.Q. Mayne, J.B. Rawlings, C.V. Rao and P.O.M. Scokaert. Constrained model predictive control: Stability and optimality. Automatica, 36(6):789-814, 2000.

M.W. McConley, B.D. Appleby, M.A. Dahleh and E. Feron. A computationally efficient Lyapunov-based scheduling procedure for control of nonlinear systems with stability guarantees. IEEE Trans. Autom. Contr., 45(1):33-49, 2000.

J.A. Primbs, V. Nevistic and J.C. Doyle. A receding horizon generalization of pointwise min-norm controllers. IEEE Trans. Autom. Contr., 45(5):898-909, 2000.

S.J. Qin and T.A. Badgwell. A survey of industrial model predictive control technology. Control Engineering Practice, 11(7):733-963, 2003.

R. Sepulchre, M. Jankovic and P. Kokotovic. Constructive nonlinear control. Springer, Berlin, Heidelberg, 1997.

E.D. Sontag. A 'universal' construction of Artstein's theorem on nonlinear stabilization. System \& control Letters, 13(2):117-123, 1989. 